\title{
Observations of the Ca II IR Triplet in High Luminosity Quasars: Exploring the Sample
}

\author{
Mary Loli Martínez-Aldama ${ }^{1, *}$, Paola Marziani ${ }^{2}$, Deborah Dultzin ${ }^{1}$, \\ Jack W. Sulentic ${ }^{3}$, Alessandro Bressan ${ }^{4}$, Yang Chen ${ }^{4}$ \\ \& Giovanna M. Stirpe \\ ${ }^{1}$ Instituto de Astronomía, Universidad Nacional Autónoma de México, \\ Aptdo. Postal 70-264, México, D.F. 04510, Mexico. \\ ${ }^{2}$ INAF, Osservatorio Astronomico di Padova, Vicolo dell Osservatorio 5, \\ 35122 Padova, Italy. \\ ${ }^{3}$ Instituto de Astronomía de Andalucía (CSIC), C/ Camino Bajo de Huetor 50, \\ 18008 Granada, Spain. \\ ${ }^{4}$ Scuola Internazionale Superiore di Studi Avanzati (SISSA), via Bonomea 265, \\ I-34136 Trieste, Italy. \\ ${ }^{5}$ INAF, Osservatorio Astronomico di Bologna, via Ranzani 1, 40127 Bologna, Italy. \\ *e-mail: maldama@astro.unam.mx
}

Received 26 June 2015; accepted 18 September 2015

DOI: $10.1007 / \mathrm{s} 12036-015-9354-9$

\begin{abstract}
We present a new spectroscopic sample of 11 quasars at intermediate redshift observed with the Infrared Spectrometer and Array Camera (ISAAC) on the ESO Very Large Telescope (VLT), covering O I $\lambda 8446$ and the $\mathrm{Ca}$ II triplet $8498,8542,8662$. The new observations - that supplement the sample presented by Martínez-Aldama et al. (2015) allow us to confirm the constraints on physical conditions and location of the region emitting the low ionization lines, as well as the relation between $\mathrm{Ca}$ II and Fe II.
\end{abstract}

Keywords. Quasars: general—quasars: emission lines—lines: profiles— star formation.

\section{Introduction}

The physical conditions for low ionization lines (LIL) emission suggest the existence of a special region within the broad line region (BLR) (Collin-Souffrin 1986; Joly 1987; Ferland \& Persson 1989; Matsuoka et al. 2007, 2008; Martínez-Aldama et al. 2015), although the properties and the origin of LILs have been an open issue for a long time. The Fe II, Ca II triplet $(\lambda 8498, \lambda 8542, \lambda 8662)$ and O I $\lambda 8446$ have been considered as representative LILs. However, the complex electronic structure of Fe II and the uncertainties in the atomic data complicate theoretical models (Netzer \& Wills 1983; Joly 1987; Collin \& Joly 2000). The O I $\lambda 8446$ and Ca II 
triplet in sources with intermediate redshift are emitted in the near-infrared region (NIR) with a strong presence of telluric bands. These difficulties notwithstanding, there are several works dedicated to the study of LILs in different AGN classes (Grandi 1980; Persson 1988; Joly 1989; Matsuoka et al. 2007, 2008; MartínezAldama et al. 2015). A close relation between Ca II and Fe II (Joly 1989) can be associated with similar physical condition for line emission $\left(n_{\mathrm{H}} \gtrsim 10^{11} \mathrm{~cm}^{-3}\right.$, $N_{\mathrm{c}} \sim 10^{25} \mathrm{~cm}^{-2}$ and $T \sim 8,000 \mathrm{~K}$ (Collin-Souffrin et al. 1980; Collin-Souffrin 1986; Ferland \& Persson 1989; Joly 1989)). The O I $\lambda 8446$ can also be emitted under these physical conditions, but it can also be emitted in a broader range of physical conditions, similar to the one of $\mathrm{H} \beta$ (Martínez-Aldama et al. 2015). The full width at half maximum (FWHM) of Ca II and O I $\lambda 8446$ are very similar and could suggest that their respective emitting regions are coupled, but the physical conditions indicate otherwise. It is convenient to imagine that the $\mathrm{O}$ I $\lambda 8446$ line may be preferentially emitted in the same region as that of $\mathrm{H} \beta$ i.e., in a system of clouds/clumps, meanwhile $\mathrm{Ca}$ II and Fe II are emitted by the accretion disk (Collin-Souffrin 1986) that provide dense gas of large column density.

Previous studies of the Ca II lines (Persson 1988; Matsuoka et al. 2007, 2008; Martínez-Aldama et al. 2015) used different selection criteria. For instance, the Persson (1988) sample was selected considering prominence of Fe II, and owing to observational complications only low redshift AGNs were observed $(0.01 \lesssim z \lesssim 0.1)$. The sample of Martínez-Aldama et al. (2015) was selected considering quasars with high luminosity $\left(-26 \gtrsim M_{\mathrm{V}} \gtrsim-29\right)$ at intermediate redshift $(0.85 \lesssim z \lesssim 1.65)$. These two samples allow for direct comparison of the behavior of the LILs at different redshifts and luminosities.

The present work extends the study of the Fe II, Ca II triplet and O I $\lambda 8446$ to 10 high luminosity and intermediate redshift quasars (section 2). The new quasars offer a more complete coverage of the Eigenvector 4DE1 sequence. Also, they constrain the region where the LILs are emitted (section 3). The new measurements confirm the relation between $\mathrm{Ca}$ II and Fe II (section 4) and the trends found previously (section 5).

\section{Sample selection and data analysis}

\subsection{Sample description}

The sample was selected considering quasars with high luminosity $\left(-27.4 \gtrsim M_{\mathrm{B}} \gtrsim\right.$ -29.0) and intermediate redshift $(0.85<z<1.68)$, where the $\mathrm{O}$ I and $\mathrm{Ca}$ II are not affected by atmospheric absorption in the infrared region. The new sample was observed with the Very Large Telescope (VLT) using the Infrared Spectrometer And Array Camera (ISAAC) during 2011. Spectra were collected with a slit width of $0.6^{\prime \prime}$ that ensured a spectral resolution of $R_{\mathrm{S}} \sim 1000$. In Table 1, the basic properties of the selected sources are reported: object name, redshift, apparent magnitude, absolute magnitude, Kellermann's coefficient for radio-loudness and spectral type according to the Eigenvector 4DE1 sequence. We also considered the spectrum around the $\mathrm{H} \beta$ region (Sulentic et al. 2004, 2006; Marziani et al. 2009). The purpose of this new sample is to extend the study of Ca II and O I $\lambda 8446$ to quasars with intermediate redshift, and to increase the statistical significance of the results 
Table 1. Basic properties of the second VLT sample.

\begin{tabular}{llcccc}
\hline Object name $^{\mathrm{a}}$ & \multicolumn{1}{c}{$z^{\mathrm{b}}$} & $m_{\mathrm{B}}^{\mathrm{c}}$ & $M_{\mathrm{B}}^{\mathrm{d}}$ & $\log R_{\mathrm{K}}^{\mathrm{e}}$ & $\mathrm{Sp.} \mathrm{T}$ \\
\hline HE0010-4050 & $0.6060 \pm 0.0004$ & 17.20 & - & - & $\mathrm{A}$ \\
HE0349-5249 & $1.5409 \pm 0.0004$ & 16.13 & -28.7 & - & $\mathrm{B} 3$ \\
HE0359-3959 & $1.5408 \pm 0.013$ & 17.09 & -27.9 & 0.22 & $\mathrm{~A} 3$ \\
HE0436-3709 & $1.4447 \pm 0.0023$ & 16.84 & -27.9 & $<0.38$ & $\mathrm{~B} 2$ \\
HE0454-4620 & $0.8528 \pm 0.003$ & 17.23 & -25.9 & 3.37 & $\mathrm{~A} 2$ \\
HE0507-3236 & $1.5770 \pm 0.0007$ & 17.36 & -27.6 & $\lesssim 0.51$ & $\mathrm{~A} 1$ \\
HE0512-3329 & $1.5873 \pm 0.0007$ & 17.03 & -28.0 & $\lesssim 0.38$ & $\mathrm{~A} 2$ \\
HE0926-0201 & $1.6824 \pm 0.0007$ & 16.23 & -29.0 & $\lesssim-0.33$ & $\mathrm{~B} 3$ \\
HE1039-0724 & $1.4584 \pm 0.0002$ & 17.16 & -27.9 & $<0.20$ & $\mathrm{~B} 1$ \\
HE1120+0154 & $1.4720 \pm 0.0004$ & 16.31 & -28.7 & -0.57 & $\mathrm{~B} 1$ \\
HE2349-3800 & $1.6040 \pm 0.0004$ & 17.5 & -27.4 & 1.93 & $\mathrm{~B} 2$ \\
\hline
\end{tabular}

\footnotetext{
${ }^{\mathrm{a} H a m b u r g / E S O}$ Survey coordinate name.

${ }^{b}$ Redshift, with uncertainty in parenthesis. Reference in Sulentic et al. $(2004,2006)$ and Marziani et al. (2009). The redshift of HE0010-4050 is determined in this work. ${ }^{\mathrm{c}}$ Apparent magnitude, reference in Sulentic et al. $(2004,2006)$ and Marziani et al. (2009).

${ }^{d}$ Absolute magnitude, reference in Sulentic et al. $(2004,2006)$ and Marziani et al. (2009).

e Decimal logarithm of the specific flux at $6 \mathrm{~cm}$ and $4440 \AA$.

${ }^{\mathrm{f}}$ Spectral type in the Eigenvector 4DE1 sequence: Population A or B.
}

obtained by Martínez-Aldama et al. (2015). Figures 1 and 2 show some examples of the the rest-frame flux and wavelength-calibrated spectra before continuum subtraction.

\subsection{Data analysis}

In the spectral range analyzed there are several features contributing to the total spectra. The main features of the optical spectral region are $\mathrm{H} \beta$, [O III] $\lambda \lambda 4959$, 5007, Fe II, Fe VI $\lambda 5160, \mathrm{Fe}$ VII $\lambda 5160$ and in some cases $\mathrm{H} \gamma$ and [O III] $\lambda 4363$. In the NIR, the main features are O I $\lambda 8446$, the Ca II triplet, Fe II, Pa9 $\lambda 9229$, the high order Paschen lines (Pa10 $\lambda$ 9014-Pa24 $\lambda 8334$ ) and Pa8 $\lambda$ 9546, Pa7 $\lambda 10050$ and [S III] $\lambda 9530$ (not always covered in our spectra). Using the task SPECFIT from IRAF (Kriss 1994), we performed multicomponent fits with all the relevant components. The fits are performed within the Eigenvector 4DE1 context (Sulentic et al. 2000, 2002; Marziani et al. 2007). The FWHM of $\mathrm{H} \beta$ are obtained by fitting to the broad component (BC) a Gaussian or a Lorentzian profile. If FWHM $(\mathrm{H} \beta)<4000 \mathrm{~km} \mathrm{~s}^{-1}$, we use a Lorentzian profile plus a blueshifted semi-broad component in some cases. If $\operatorname{FWHM}(\mathrm{H} \beta) \geq 4000 \mathrm{~km} \mathrm{~s}^{-1}$ we used a double Gaussian profile, with one of them being a very broad component (VBC) (Marziani et al. 2009). The FWHM for $\mathrm{BC}$ and VBC shifts and ratios are the same for the $\mathrm{H} \beta$ line and Paschen lines. For each spectral region, we set a local continuum come from the accretion disk emission. We cannot find any effect from the host galaxy continuum. Since the Ca II triplet comes from an optically thick transition (Ferland \& Persson 1989), the three lines have the same intensity. Also, we do not consider any shift in the rest-frame wavelength. The Fe II template used in the optical range was set up by Marziani et al. (2009). In the NIR, we apply the theoretical or semi-empirical template developed by Garcia-Rissmann et al. (2012). We select the template according 

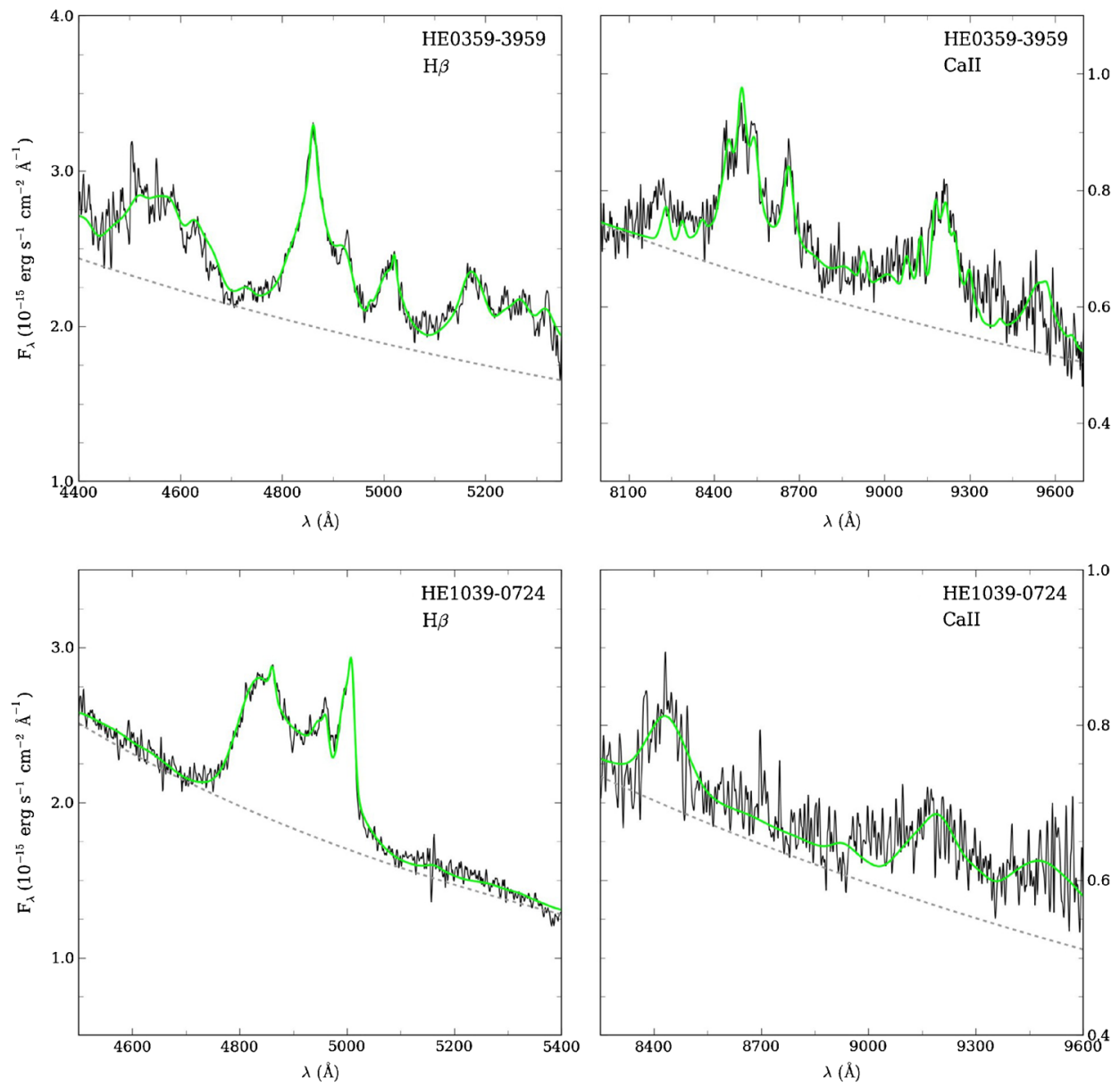

Figure 1. Calibrated spectra with the continuum contribution for $\mathrm{H} \beta$ (left panel) and Ca II (right panel) regions for HE0359-3959 and HE1039-0724. The continuous green line marks the best fit and dashed grey line indicates the continuum level. Abscissae are rest-frame wavelength in $\AA$ and ordinates are rest-frame specific flux in units of $10^{-15} \mathrm{erg} \mathrm{s}^{-1} \mathrm{~cm}^{-2} \AA^{-1}$.

to the best $\chi^{2}$ minimization. In practice, we fit the theoretical template to all quasar spectra, although there are many Fe II transitions that this template cannot reproduce.

\section{Exploring the sample}

\subsection{Behavior of low ionization lines inside the Eigenvector 4DE1 scheme}

Quasars of the first sample identified a distinct $\mathrm{Ca}$ II behavior for population $\mathrm{A}$ and $\mathrm{B}$ objects. They also confirmed a relation between $\mathrm{Ca}$ II and Fe II strength. In the present sample, there are two sources that make particularly evident the behavior of the low ionization lines within the context of the Eigenvector 4DE1 diagram. These quasars are HE0359-3959 and HE1039-0724. HE0359-3959 is a population 


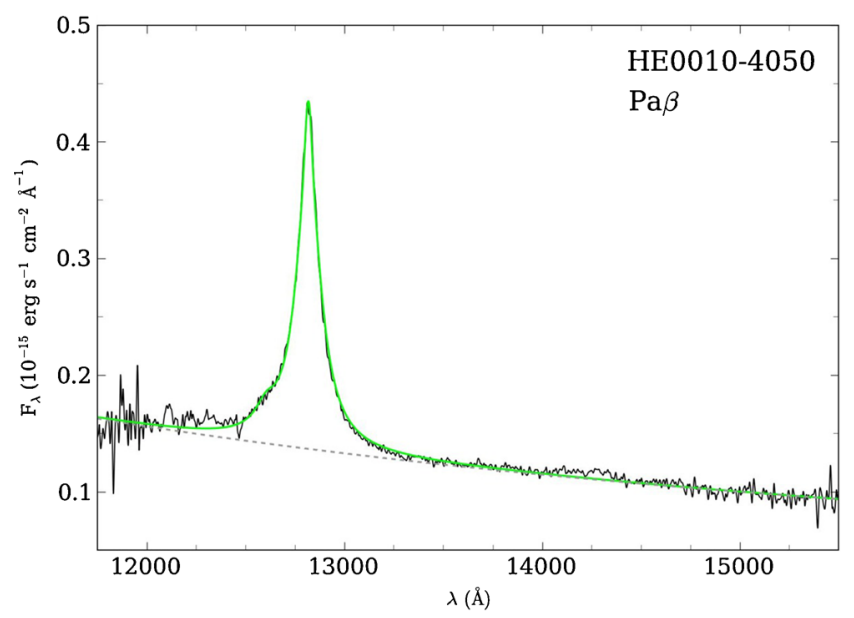

Figure 2. HE0010-4050 calibrated spectrum for the region around $\mathrm{Pa} \beta \lambda 12818$. The continuous green line marks the best fit, and the dashed grey line marks the continuum level. Abscissae are rest-frame wavelength in $\AA$ and ordinates are rest-frame specific flux in units of $10^{-15} \mathrm{erg}$ $\mathrm{s}^{-1} \mathrm{~cm}^{-2} \AA^{-1}$.

A3 object, and it shows a strong Fe II emission. The NIR spectrum also shows a strong $\mathrm{Ca}$ II emission. It was the first time that we could observe the $\mathrm{Ca}$ II so intense and partially resolved in a quasar at intermediate redshift. In some objects, the Ca II triplet presence could be detected through the $\lambda 8662$ line, while the other two lines are totally blended. The $\lambda 8498$ and $\lambda 18542$ lines are clearly identified in HE03593959. In this quasar, some Fe II emissions were clearly observed, for example the emission at the blue side of O I $\lambda 8446$ and the bump at $\sim 9400 \AA$. There was no evidence of the [S III] $\lambda$ 9530, and neither of the optical forbidden narrow lines. Due to the strength of Fe II, this quasar can be used as a proxy, similar to IZw1, to improve the Fe II templates in the NIR.

The quasar that represents the population B is HE1039-0724. This object showed a very large width and strong $\mathrm{H} \beta$ emission. The $\mathrm{BC}$ of $\mathrm{H} \beta$ was blueshifted and the intensity of the VBC was almost two times the intensity of the BC. Also, it showed a strong blueshifted [O III] $\lambda \lambda 4959,5007$ emission. The Fe II emission was very small, and this was reflected in the behavior of the Ca II. The bump O I $+\mathrm{Ca}$ II could be almost reproduced only with the O I profile. These objects clearly revealed the relationship between Ca II and Fe II, and supported a similarity between the physical emission conditions of both lines (Joly 1989; Matsuoka et al. 2007; Martínez-Aldama et al. 2015). On the other hand, in the Eigenvector 4DE1 context, low ionization lines, such as Ca II and Fe II showed a variation along the sequence: from strong Fe II or Ca II emission in population A objects, to a minimum emission in population B objects (Negrete et al. 2012).

\subsection{A new look of the emitting region of $O \mathrm{I} \lambda 8446$}

HE0454-4620 is a blazar cataloged as a flat-spectrum radio quasar (Massaro et al. 2009). The existence of the broad lines indicates that the line-of-sight is not highly 
misaligned to the jet, in agreement to the standard radio-loud unification (Blandford \& Rees 1978; Urry \& Padovani 1995). In the optical spectrum of HE0454-4620, we can observe $\mathrm{H} \beta$ emission and a prominent [O III] $\lambda \lambda 4959,5007$ contribution. Also, the continuum shows a positive slope, which is common in blazars (for example, Ruan et al. 2014). According to the FWHM of $\mathrm{H} \beta$, this object belongs to A2 population with a small Fe II emission. This blazar also corroborate the hypothesis that a low Fe II emission implies a very low Ca II contribution. A Ca II contribution is not even necessary for this object. This behavior is not followed by $\mathrm{O}$ I or $\mathrm{H} \beta$. This supports the view that $\mathrm{O} I$ is preferentially emitted in a region, at least in part, different from the one emitting $\mathrm{Ca}$ II, and the possibility that $\mathrm{O}$ I $\lambda 8446$ is emitted by a configuration of clouds/clumps like $\mathrm{H} \beta$, rather than by the accretion disk that provides a dense low-ionization medium for Ca II and Fe II.

\subsection{HE0010-4050, determination of a new redshift value}

The spectrum of HE0010-4050 only presents an isolated emission that could be associated with the $\mathrm{O}$ I $\lambda 8446$. Assuming that the redshift value is $z=1.5$ (Savage $\&$ Wright 1981), the O I $\lambda 8446$ line shows a blueshift $\approx 7650 \mathrm{~km} \mathrm{~s}^{-1}$. Unfortunately, the $H \beta$ spectrum has a very low signal-to-noise $(\mathrm{S} / \mathrm{N})$ ratio and hence we cannot distinguish any spectral feature, as a result it is not possible to determine if the blueshift is real. On other hand, in the near-infrared region there are two main features: O I + Ca II and Fe II + Pa9 $\lambda 9229$ bumps. In the spectrum of HE0010-4050 the expected contribution is not present (see Fig. 2). This led us to think that there is an error in the previous redshift determination. The redshift of this quasar was computed using a photometric plate (Savage \& Wright 1981) and it was picked by the surveys where the quasar has been included without ensuring that its redshift is correct. According to the spectra reported by Glikman et al. (2006) and Landt et al. (2008, 2011), the region observed is in agreement with the region around $\mathrm{Pa} \beta$ $\lambda 12818$ where only the contribution of this line is presented. Using $\mathrm{Pa} \beta \lambda 12818$ line as a rest-frame, the redshift of this quasars is $z \approx 0.606 \pm 0.0004$ at $1 \sigma$ confidence.

\section{Results from our enlarged sample of quasars}

\subsection{O I, Ca II and Fe II relations}

The present sample of 11 quasars complements our own as well as other previous sample and results (Persson 1988; Joly 1989; Ferland \& Persson 1989; MartínezAldama et al. 2015). The object HE0454-4620 is not considered since it does not show $\mathrm{Ca}$ II emission. The left panel of Fig. 3 shows the ratios $\mathrm{Ca} \mathrm{II} / \mathrm{H} \beta$ and $\mathrm{Fe}$ II $\lambda 4570 / \mathrm{H} \beta$, indicating that these line ratios are correlated and suggesting that both $\mathrm{Ca}$ II and Fe II are emitted in the same region, in agreement with the result of Joly (1989) models, where the Ca II and Fe II intensities increase at high density and low temperature. The extreme population A and B quasars described in section 3.1 are located at the borders of the trend, showing the behavior of the low ionization lines. The FWHM of Ca II and O I are very similar and slightly narrower than the FWHM of $\mathrm{H} \beta$, indicating that $\mathrm{Ca}$ II and $\mathrm{O}$ I may be emitted at a slightly larger emissivityweighted distance with respect to $\mathrm{H} \beta$. However, this does not mean that $\mathrm{O}$ I and $\mathrm{Ca}$ II are emitted at the same place. Photoionization models suggest (Martínez-Aldama 

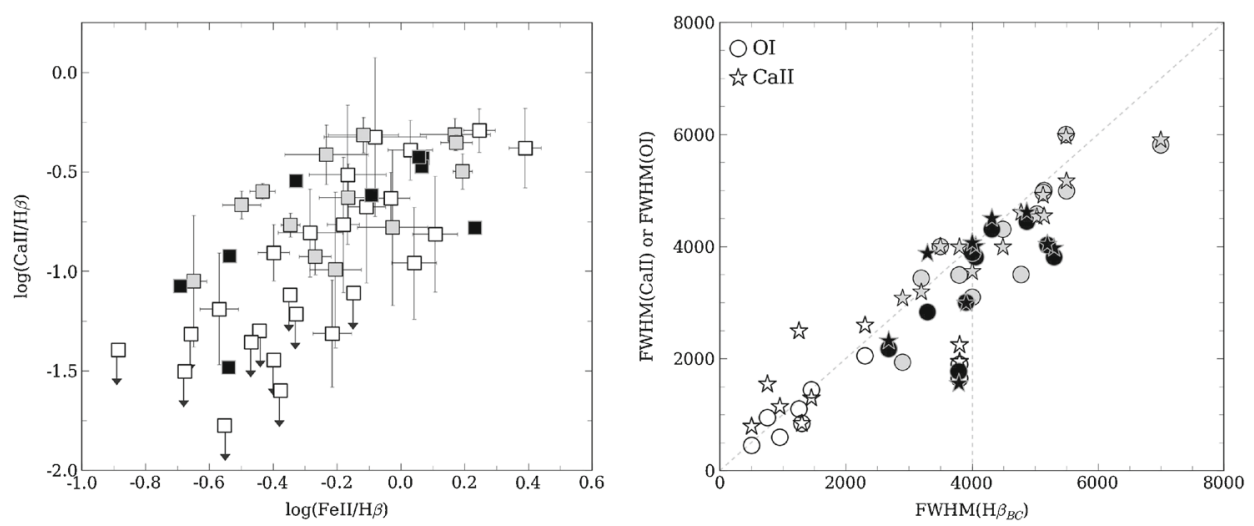

Figure 3. Left panel: Relation between the observed ratios $\log (\mathrm{Fe}$ II $\lambda 4570 / \mathrm{H} \beta)$ and $\log (\mathrm{Ca}$ $\mathrm{II} / \mathrm{H} \beta)$. The open symbols correspond to the sample of Persson (1988), the filled grey corresponds to the sample of Martínez-Aldama et al. (2015) and the filled black ones to the sample presented in this work. Right panel: FWHM behavior for $\mathrm{H} \beta$, O I (circles) and Ca II (stars). The meaning of the line coding is the same as in the left panel. The diagonal dashed line is the equality line. The vertical dashed line at $4000 \mathrm{~km} \mathrm{~s}^{-1}$ indicates the separation between Pop. A and B.

et al. 2015) a wide density range over which $\mathrm{O}$ I can be emitted. Considering the $\mathrm{O}$ $\mathrm{I}$ and $\mathrm{H} \beta$ behavior in HE0454-4620, we can conclude that only $\mathrm{O}$ I is preferentially emitted in a system of clouds where $\mathrm{H} \beta$ is also emitted.

\subsection{Starbursts in intermediate redshift quasars?}

Our sample as well as Persson's (1988) sample contain objects with different properties: luminosity, redshift, Fe II or Ca II intensity and profiles. When we compare
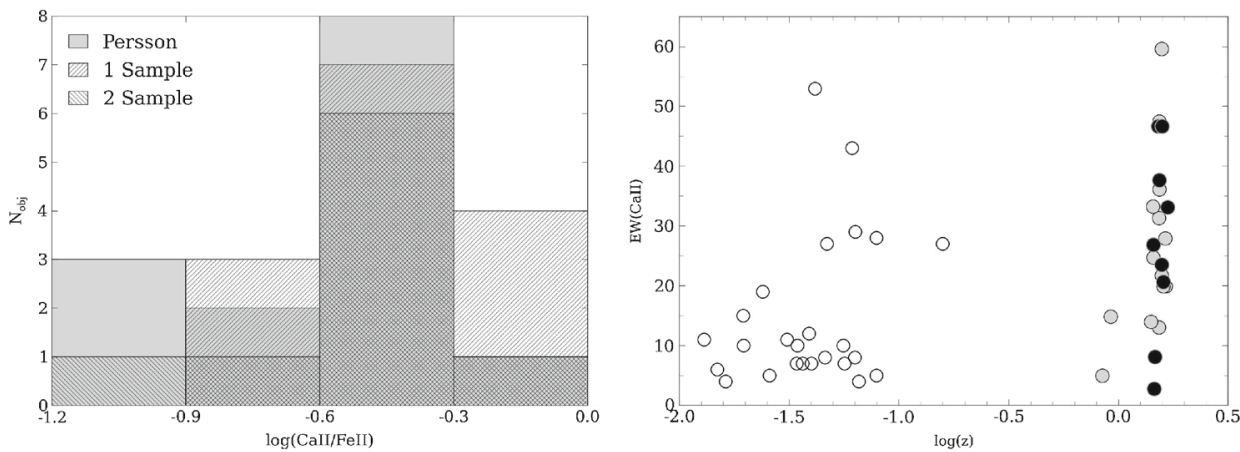

Figure 4. Left panel: Distribution of $\mathrm{Ca}$ II/Fe II $\lambda 4570$ with the newest measurements (1 Sample), Martinez-Aldama et al.'s (2015) sample (2 Sample), and Persson (1988) objects (grey shaded). Right panel: Behavior of the equivalent width of Ca II with respect to redshift. The open circles indicate the Persson's sample, filled circles correspond to Martinez-Aldama et al.'s (2015) sample, and black circles indicate the sample analyzed in this work. 
the intensity of the Ca II with respect to Fe II, we find that the objects at intermediate redshift show a slight overabundance (Fig. 4, left panel). The same effect is observed when we plot the equivalent width of Ca II as a function of redshift (Fig. 4, right panel). The new data confirms the results of Martínez-Aldama et al. (2015). A straightforward interpretation is that the broad line emitting gas has been enriched by a recent star formation burst. The Ca II is an $\alpha$ element; it is produced through the core collapse supernovae on timescales $\lesssim 3 \times 10^{7}$ yr. If the iron nucleosynthesis is mainly associated with type Ia supernovæ on timescales of $\sim 10^{9} \mathrm{yr}$ (Wyse \& Gilmore 1988), the enhanced Ca II may imply recent star formation in intermediate $z$ quasars. However, the $\mathrm{Ca}$ II/Fe II ratio is not uniformly distributed over the luminosity and redshift range, and can be affected by biases in luminosity and redshift, so that the conclusion is tentative at the moment. An investigation involving iron yields from type II supernovæ is planned.

\section{Conclusions}

The new sample provides additional data that confirm the relation between Ca II and Fe II, as well as the trends within the Eigenvector 4DE1 scheme. If Fe II is strong, $\mathrm{Ca}$ II is also strong, and vice versa. This is the case of HE0359-3959, an A3 object where Fe II and Ca II are both very strong. In this object, an unblended Ca II triplet can be observed. HE1039-0724, a B1 object, shows a very small contribution of Fe II and Ca II. These two quasars represent the extremes in the range of LIL intensity along the Eigenvector 4DE1 sequence. On the other hand, the blazar HE0454-4620 shows a little Fe II emission, no $\mathrm{Ca}$ II, and strong $\mathrm{O}$ I $\lambda 8446$. This indicates that $\mathrm{O}$ I $\lambda 8446$ is also emitted in other parts of the BLR, probably where part of $\mathrm{H} \beta$ is also emitted. We confirm the overabundance of $\mathrm{Ca}$ II in the quasars at intermediate redshift, that it could be related with a recent burst of star formation.

\section{Acknowledgements}

This work is based on observations collected at the European Organisation for Astronomical Research in the Southern Hemisphere, Chile, under programme ID 086.B-0300(A).

\section{References}

Blandford, R. D., Rees, M. J. 1978, in: BL Lac Objects, edited by A. M. Wolfe, 328

Collin, S, Joly, M. 2000, NAR, 44, 531.

Collin-Souffrin, S. 1986, A\&A, 166, 115.

Collin-Souffrin, S., Joly, M., Dumont, S., Heidmann, N. 1980, A\&A, 83, 190.

Ferland, G. J, Persson, S. E. 1989, ApJ, 347, 656.

Garcia-Rissmann, A., Rodríguez-Ardila, A., Sigut, T. A. A., Pradhan, A. K. 2012, ApJ, $751,7$.

Glikman, E., Helfand, D. J., White, R. L. 2006, ApJ, 640, 579.

Grandi, S. A. 1980, ApJ, 238, 10.

Joly, M. 1987, $A \& A, \mathbf{1 8 4}, 33$.

Joly, M. 1989, A\&A, 208, 47.

Kriss, G. 1994, in: Astronomical Society of the Pacific Conference Series, Vol. 61, Astronomical Data Analysis Software and Systems III, edited by D. R. Crabtree, R. J. Hanisch \& J. Barnes, 437. 
Landt, H., Bentz, M. C., Ward, M. J., Elvis, M., Peterson, B. M., Korista, K. T, Karovska, M. 2008, ApJS, 174, 282.

Landt, H., Elvis, M., Ward, M. J., Bentz, M. C., Korista, K. T, Karovska, M. 2011, MNRAS, 414, 218.

Martínez-Aldama, M. L., Dultzin, D., Marziani, P., Sulentic, J. W., Bressan, A., Chen, Y., Stirpe, G. M. 2015, ApJS, 217, 3.

Marziani, P., Sulentic, J. W., Stirpe, G. M., Zamfir, S., Calvani, M. 2009, A\&A, 495, 83.

Massaro, E., Giommi, P., Leto, C., Marchegiani, P., Maselli, A., Perri, M., Piranomonte, S., Sclavi, S. 2009, A\&A, 495, 691.

Matsuoka, Y., Oyabu, S., Tsuzuki, Y., Kawara, K. 2007, ApJ, 663, 781.

Matsuoka, Y., Peterson, B. A., Oyabu, S., Kawara, K., Asami, N., Sameshima, H., Ienaka, N., Nagayama, T., Tamura, M. 2008, ApJ, 685, 767.

Negrete, C. A., Dultzin, D., Marziani, P., Sulentic, J. W. 2012, ApJ, 757, 62.

Netzer, H, Wills, B. J. 1983, ApJ, 275, 445.

Persson, S. E. 1988, ApJ, 330, 751.

Ruan, J. J., Anderson, S. F., Plotkin, R. M., Brandt, W. N., Burnett, T. H., Myers, A. D, Schneider, D. P. 2014, ApJ, 797, 19.

Savage, A, Wright, A. E. 1981, MNRAS, 196, 927.

Sulentic, J. W., Bachev, R., Marziani, P., Negrete, C. A., Dultzin, D. 2007, ApJ, 666, 757.

Sulentic, J. W., Marziani, P., Dultzin-Hacyan, D. 2000, ARAA, 38, 521.

Sulentic, J. W., Marziani, P., Zamanov, R., Bachev, R., Calvani, M., Dultzin- Hacyan, D. 2002, ApJL, 566, L71.

Sulentic, J. W., Repetto, P., Stirpe, G. M., Marziani, P., Dultzin-Hacyan, D., Calvani, M. 2006, $A \& A, \mathbf{4 5 6}, 929$.

Sulentic, J. W., Stirpe, G. M., Marziani, P., Zamanov, R., Calvani, M., Braito, V. 2004, A\&A, 423, 121.

Urry, C. M., Padovani, P. 1995, PASP, 107, 803.

Wyse, R. F. G., Gilmore, G. 1988, AJ, 95, 1404. 